Proceedings of the 9th Workshop on Quantum Chaos and Localisation Phenomena, May 24-26, 2019, Warsaw, Poland

\title{
Investigation of the Elastic Enhancement Factor in Microwave Chaotic Cavities in the Presence of Significantly Opened Channels
}

\author{
M. Bialous ${ }^{a}$, B. Dietz ${ }^{b}$ AND L. Sirko ${ }^{a}$ \\ ${ }^{a}$ Institute of Physics, Polish Academy of Sciences, Aleja Lotnikow 32/46, PL-02668 Warsaw, Poland \\ ${ }^{b}$ School of Physical Science and Technology, and Key Laboratory for Magnetism and Magnetic Materials of MOE, \\ Lanzhou University, Lanzhou, Gansu 730000, China
}

\begin{abstract}
The elastic enhancement factor $F$ is commonly used to study the relationship between elastic and inelastic scattering processes. In chaotic scattering the elastic processes are known to be enhanced over the inelastic ones. Most thorough experimental studies of $F$ are possible by using microwave setups simulating the quantum systems. In this paper the elastic enhancement factor $F_{M}^{(1)}\left(\gamma^{\text {tot }}\right)$ is studied in a microwave chaotic quarter bow-tie cavity emulating a chaotic two-dimensional quantum billiard with preserved time reversal symmetry in the presence of significantly opened channels $M$ with the total absorption strength $\gamma^{\text {tot }}$. The experimental results are obtained for $2 \leq M \leq 9$ open channels characterized by the average transmission coefficient $0.34<T<0.98$, moderate internal absorption strength $\gamma=0.9-2.8$, and the total absorption strength $\gamma^{\text {tot }}=1.6-11.6$. We show that the experimental results are close to the theoretical predictions. Moreover, the spectral properties of the microwave quarter bow-tie billiard are studied for $M=2$ channels using missing level statistics. They comply with those of generic time reversal invariant and classically fully chaotic systems.
\end{abstract}

DOI: 10.12693/APhysPolA.136.765

PACS/topics: 05.45.Mt, 03.65.Nk

\section{Introduction}

The elastic enhancement factor $F$ was introduced more than half a century ago by Moldauer [1] and subsequently frequently considered in nuclear physics [2-5] and in other fields [6-9]. From the experimental point of view the elastic enhancement factor $F$ is especially interesting because it can be used to study realistic open systems possessing preserved or broken time reversal symmetry. Its properties have been studied in several precisely controllable systems such as microwave cavities [8, 10-14] and networks [15-19]. Model systems such as microwave networks [18, 20-23] simulating quantum graphs, flat microwave cavities simulating twodimensional quantum billiards [24-29] and experiments with the Rydberg atoms strongly driven by microwave fields [30-36] were very successfully used to simplify analysis of complex systems. For example, quantum graphs can be used to model such complicated systems as experimental setups to realize high-dimensional multipartite quantum states [37], functional connectivity in preclinical Alzheimer's disease [38], and discrete-time quantum gravity models [39]. The universality of the elastic enhancement factor $F$ has been also tested in the wave scattering experiments using microwave cavities $[8,11]$ in the presence of absorption. Systems with time reversal symmetry have been tested by Zheng et al. [11]. Dietz et al. [8] have studied the universality of the elastic

corresponding author enhancement factor $F$ with microwave cavities in the case of preserved and partially broken time reversal symmetries. Quite recently an extensive study of the elastic enhancement factor $F$ for microwave cavities with time reversal symmetry in a low absorption regime has been published by Yeh et al. [12].

\section{Theory}

Furthermore, the elastic enhancement factor $F$ has been also studied for microwave irregular networks [20] with preserved and broken time reversal symmetry in the case of moderate and large absorption strength $5 \leq \gamma^{\prime} \leq 54.4[15-17,19]$. The absorption strength $[6,7]$ is defined as follows: $\gamma^{\prime}=2 \pi \Gamma / \Delta$, where $\Gamma$ is the absorption width and $\Delta$ is the mean level spacing. Microscopically, $\gamma^{\prime}$ can be modeled by means of a large number of weakly open, coupled to continuum, parasitic channels [7]. Such an approximation is often called the Verbaarschot regime [3, 4]. Reference [5] deals with the concept of open systems with a transient from regular to chaotic internal dynamics. There the openness $\eta$ is introduced instead of the absorption strength $\gamma^{\prime}$, which is described formally by the same formula. For such systems $\Gamma$ is the well-known Weisskopf width and the reciprocal of $\Gamma$ is the time that the incoming particle spends on average inside the system. The situation changes considerably if additionally too many weakly open channels have to deal with several significantly open channels. Such a situation was considered theoretically in Ref. [9] and can be experimentally realized in microwave experiments. Under the mentioned conditions the absorption 
strength $\gamma^{\prime}$ has to be supplemented by additional parameters connected with the open channels $M$. Therefore, the enhancement factor depends on the number of open channels $M$, the average transmission coefficient $T$ of the channels, and the internal absorption strength $\gamma$, which as in the case of the Verbaarschot regime can be modelled by means of many weakly open channels.

Such a generalized elastic enhancement factor has not been studied experimentally as a function of the number of open channels $M$ yet. In the present paper we report on the results of experimental studies of the elastic enhancement factor $F_{M}^{(1)}\left(\gamma^{t o t}\right)$ [9] using a microwave chaotic quarter bow-tie cavity simulating a chaotic twodimensional (2D) quantum billiard with preserved time reversal symmetry in the presence of open channels $M$ and the total absorption strength $\gamma^{\text {tot }}$. The experimental results in the frequency range $\nu=6-12 \mathrm{GHz}$ were obtained for $2 \leq M \leq 9$ open channels, internal absorption strength $\gamma=0.9-2.8$, and the total absorption strength $\gamma^{t o t}=1.6-11.6$. The total absorption strength of the cavity in the presence of $M$ open channels and internal absorption $\gamma$ is given by $\gamma^{\text {tot }}=M T+\gamma$. Here, we assume that the channels $M$ are equivalent and characterized by the average transmission coefficient $T=\frac{1}{M} \sum_{i=1}^{M} T_{i}$. This assumption was experimentally verified. All $T_{i}$ in the investigated frequency range $\nu=6-12 \mathrm{GHz}$ were within $5 \%$ to each other. Compared to Ref. [14] our analysis also includes the case of $M=2$ channels.

In the case of the two-port scattering matrix

$$
\hat{S}(\nu)=\left[\begin{array}{cc}
S_{a a}(\nu) & S_{a b}(\nu) \\
S_{b a}(\nu) & S_{b b}(\nu)
\end{array}\right],
$$

the elastic enhancement factor $F_{M}^{(1)}\left(\gamma^{\text {tot }}\right)$ is defined by the following relation [8]:

$F_{M}^{(1)}\left(\gamma^{\text {tot }}\right)=\frac{\sqrt{\left\langle\left|S_{a a}^{f l}(\nu)\right|^{2}\right\rangle\left\langle\left|S_{b b}^{f l}(\nu)\right|^{2}\right\rangle}}{\langle|\left(\left.S_{a b}^{f l}(\nu)\right|^{2}\right\rangle}=\frac{\sqrt{C_{a a}(0) C_{b b}(0)}}{C_{a b}(0)}$,

where the fluctuating part of the two-port scattering matrix element $S_{a b}^{f l}(\nu)=S_{a b}(\nu)-\left\langle S_{a b}(\nu)\right\rangle$ and $C_{a b}(0)$ denotes the $S$-matrix two-point correlation function

$$
C_{a b}(\epsilon)=\left\langle S_{a b}^{f l}(\nu) S_{a b}^{f l *}(\nu+\epsilon)\right\rangle,
$$

calculated at $\epsilon=0$.

For strong internal absorption $\gamma \gg 1$ or a large number of open strong channels $M \gg 1$ the enhancement factor should saturate to $F_{M}^{(1)}\left(\gamma^{\text {tot }}\right)=2$, see $[6,7,9,11]$.

\section{Experimental}

In the experiment we used a microwave chaotic quarter bow-tie cavity to simulate a two dimensional (2D) chaotic quantum billiard. If the excitation frequency $\nu$ is below $\nu_{\max }=c / 2 d$, where $c$ is the speed of light in the vacuum and $d$ is the height of the cavity, only the transverse magnetic modes can be excited inside the cavity. Then, the Helmholtz equation of flat microwave cavity and the Schrödinger equation of a quantum billiard of

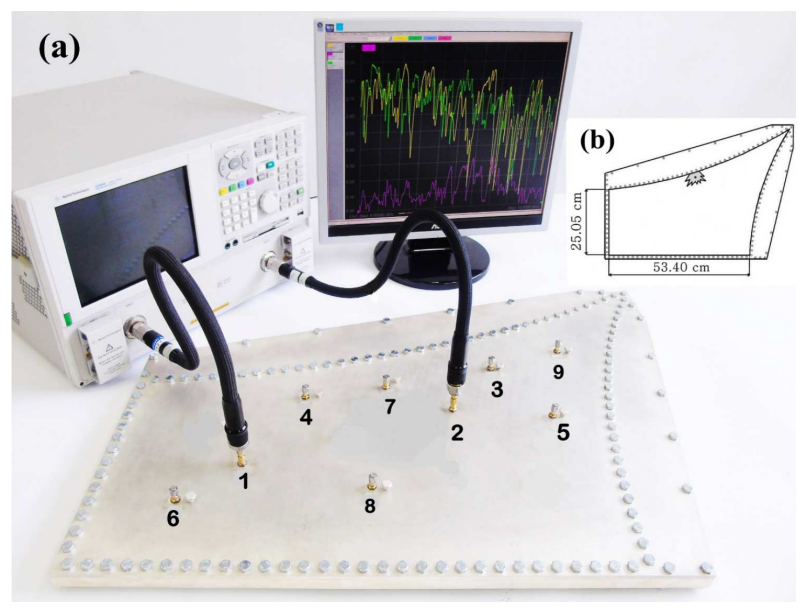

Fig. 1. (a) Photo of the experimental setup. The scattering matrix $\hat{S}(\nu)$ of the cavity was measured in the frequency window 6-12 GHz. The vector network analyzer Agilent E8364B was connected to the microwave antennas through the flexible microwave cables (holes $A_{1}$ and $A_{2}$ ). The measuring antennas with pin diameter $0.9 \mathrm{~mm}$ and length $5.8 \mathrm{~mm}$ were protruded through holes into the cavity introducing $M=2$ open channels. The additional $M^{\prime}$ open channels were introduced to the cavity by using $M^{\prime}$ antennas having the same size as the measuring ones but shunt with $50 \Omega$ loads. (b) The scheme of the quarter bow-tie microwave cavity used for measuring of the two-port scattering matrix $\hat{S}(\nu)$. In order to create different realizations of the cavity a metallic perturber was moved inside the cavity.

corresponding shape are identical [40]. Figure 1 shows a photography of the microwave chaotic quarter bowtie cavity, which was used for the measurement of the two-port scattering matrix $\hat{S}$. The size of the cavity was the following: the area of the bottom (top) plate $A=1828.5 \mathrm{~cm}^{2}$, the perimeter $L=202.3 \mathrm{~cm}$, and the height $h=1.2 \mathrm{~cm}$. The cavity was made of polished aluminium type EN 5754. The inner surface was entirely covered by $20 \mu \mathrm{m}$ layer of silver, which leads to a reduction of the internal absorption by around $30 \%$. The cavity was constructed out of two pieces: the bottom plate, which was integrated with the side walls and the top plate containing 9 randomly distributed holes $A_{1}$, $A_{2}, \ldots, A_{9}$ (see Fig. 1). Both elements were very tightly squeezed together using 127 screws. The quality factor $Q$ of the cavity ranged between 2000 and 3000 .

The scattering matrix $\hat{S}$ was measured in the frequency range 6-12 GHz. The higher range of the frequency was limited by the cut-off frequency of the cavity $\nu_{\max }=$ $c / 2 d \simeq 12.49 \mathrm{GHz}$. The measurements of the two-port scattering matrix $\hat{S}$ were performed using an Agilent E8364B microwave vector network analyzer (VNA) which was coupled to the cavity through the HP 85133-616 and HP 85 133-617 flexible microwave cables via the two measuring antennas. The measuring antennas with the pin diameter $0.9 \mathrm{~mm}$ and the length $5.8 \mathrm{~mm}$ were protruded through the holes into the cavity introducing $M=2$ open channels. The additional $M^{\prime}$ open channels required for 
the measurement of the enhancement factor $F_{M}^{(1)}\left(\gamma^{\text {tot }}\right)$ with $M>2$ were introduced to the cavity by $M^{\prime}$ antennas, which had the same size as the measuring ones, but were shunt with $50 \Omega$ loads. The metallic perturber "half-star" with the area $A \simeq 9 \mathrm{~cm}^{2}$ and the perimeter $L \simeq 26 \mathrm{~cm}$ was inserted into the cavity (see part (b)) to obtain 100 different realizations of the cavity. It was moved and slightly rotated alongside the walls quite far from the antennas using an external magnet.

Internal absorption $\gamma$ of the cavity can be changed by varying the frequency range of the measurements or more effectively by the application of microwave absorbers. We were interested in the smallest possible absorption, which can be varied by the choice of the microwave frequency range.

\section{Results and discussion}

The internal absorption strength $\gamma$ of the cavity was evaluated by adjusting the theoretical distributions of the reflection coefficient $P\left(r_{j}\right)$ of the diagonal elements $S_{j j}(\nu)=\sqrt{r_{j}} e^{i \theta_{j}}$ of the scattering matrix $\hat{S}(\nu)$ to the experimental ones [14]. The index $j=a, b$ denotes the port $a$ or $b$, and $r_{j}$ and $\theta_{j}$ are the reflection coefficient and the phase evaluated at the $j$-th port of the cavity. For chaotic systems with preserved time reversal symmetry $(\beta=1)$ the distribution $P\left(r_{j}\right)$ of the reflection coefficient $r_{j}$ can be evaluated from the distribution $P\left(x_{j}, \theta_{j}\right)$ given by the formula (7) in Ref. [14]. In this formula, which can be also applied for the cases with many open channels, $x_{j}=\frac{1+r_{j}}{1-r_{j}}$.

The influence of the open channels on the spectral properties of microwave cavities were investigated based on missing level statistics where we considered the nearest-neighbor spacing distribution $P(s)[41,42]$ and the average power spectrum $\langle s(\tilde{k})\rangle[43]$. The system with missing levels is characterized by the fraction of observed levels $\varphi$. The distribution $P(s)$ for the microwave quarter bowtie cavity with two open channels $M=2$ is shown in Fig. 2a (bars). The distribution $P(s)$ was averaged over 15 microwave cavity configurations. In the calculations 3150 eigenfrequencies of the cavity were used. Figure 2a shows that the experimental distribution $P(s)$ is in very good agreement with the nearestneighbor spacing distribution $P(s)$ (dash-dotted line) accounting for missing levels with the fraction of observed levels $\varphi=0.94$, which was found in the experiment. Because the fraction of observed levels $\varphi$ is close to 1 , $P(s)$ is also very close to the Wigner distribution (solid line), which is characteristic for chaotic systems with preserved time reversal symmetry.

For a more detailed analysis of the degree of chaoticity of the measured system we used the power spectrum of the deviation of the $q$-th nearest-neighbor spacing from its mean value $q, \delta_{q}=\epsilon_{q+1}-\epsilon_{1}-q[18,41,43,44]$. For a sequence of $N$ levels it is given in terms of the Fourier spectrum from "time" $q$ to $k, S(k)=\left|\tilde{\delta}_{k}\right|^{2}$, with $\tilde{\delta}_{k}=\frac{1}{\sqrt{N}} \sum_{q=0}^{N-1} \delta_{q} \exp (-(2 \pi i k q) / N)$. It was shown
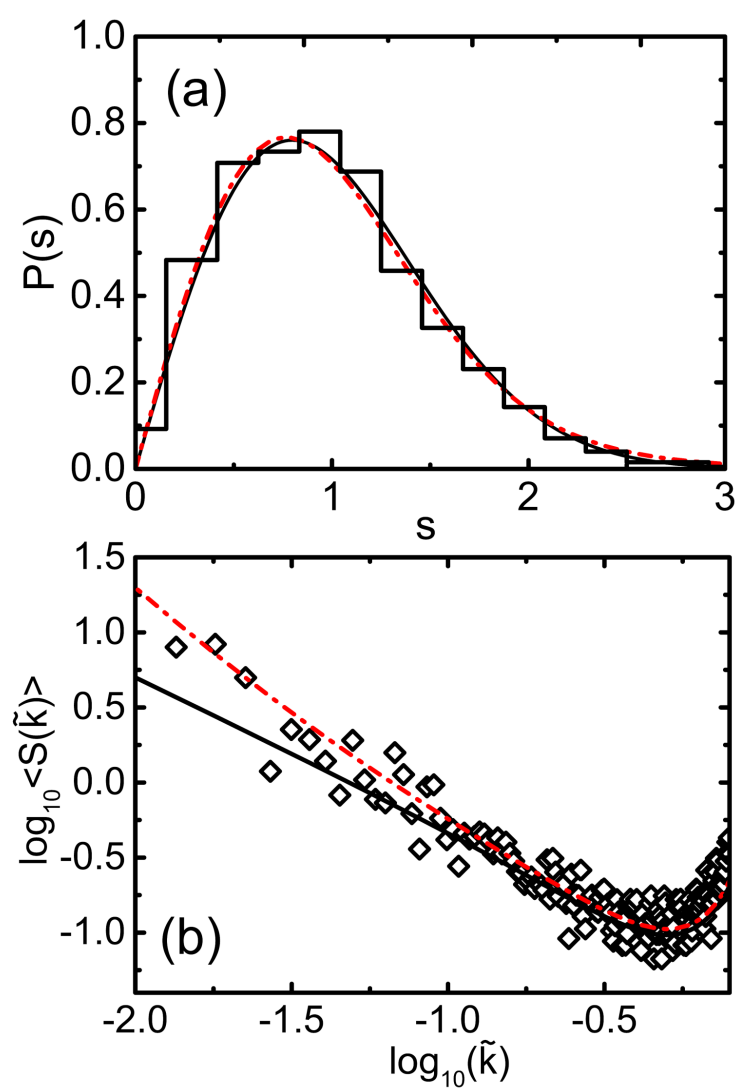

Fig. 2. (a) The nearest-neighbor spacing distribution $P(s)$ (bars) measured for the microwave quarter bow-tie cavity for two open channels $M=2$. The experimental distribution is compared to the theoretical distribution $P(s)$ (red dash-dotted line) accounting for missing levels with the fraction of observed levels $\varphi=0.94$. The theoretical prediction for the $\operatorname{GOE}(\varphi=1)$ is shown as solid line. (b) The experimental distribution of the average power spectrum $\langle s(\tilde{k})\rangle$ (black diamonds) is compared to the theoretical prediction (red dash-dotted line). The fraction of the observed levels was determined to be $\varphi=0.94 \pm 0.02$. The experimental results are also compared to the theoretical prediction for $\operatorname{GOE}(\varphi=1)$ (black solid line).

in Refs. [45, 46], that for $\tilde{k}=k / N \ll 1$ the power spectrum exhibits a power law dependence $\langle S(\tilde{k})\rangle \propto \tilde{k}^{-\alpha}$. Here, for chaotic systems $\alpha=1$, independently of whether time reversal invariance is preserved or not, and $\alpha=2$ for regular systems. In the case of missing levels the power spectrum $\langle s(\tilde{k})\rangle$ is given by the formula

$$
\begin{aligned}
& \langle s(\tilde{k})\rangle=\frac{\varphi}{4 \pi^{2}}\left[\frac{K(\varphi \tilde{k})-1}{\tilde{k}^{2}}+\frac{K(\varphi(1-\tilde{k}))-1}{(1-\tilde{k})^{2}}\right] \\
& \quad+\frac{1}{4 \sin ^{2}(\pi \tilde{k})}-\frac{\varphi^{2}}{12} .
\end{aligned}
$$

Here, $0 \leq \tilde{k} \leq 1$. $K(\tau)$ is the spectral form factor, which for $\tau \leq 1$ equals $K(\tau)=2 \tau-\tau \log (1+2 \tau)$ for the Gaussian orthogonal ensemble (GOE) in the random 


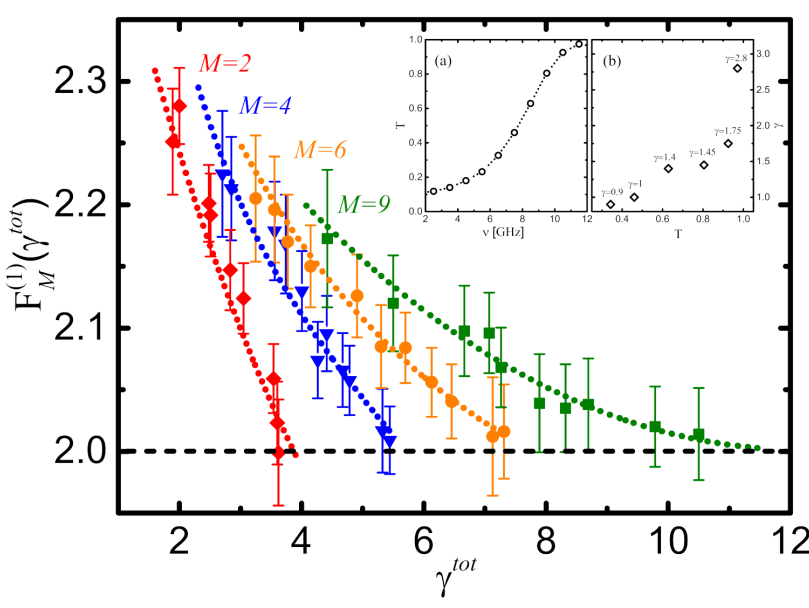

Fig. 3. The elastic enhancement factor $F_{M}^{(1)}\left(\gamma^{t o t}\right)$ of the two-port scattering matrix $\hat{S}$ of the microwave quarter bow-tie cavity for $M=2$ (red full diamonds), $M=4$ (blue full triangles), $M=6$ (orange full circles), and $M=9$ (green full squares) open channels as a function of the total absorption strength $\gamma^{\text {tot }}$. The theoretical results for $M=2, M=4, M=6$, and $M=9$ channels are shown with red, blue, orange, and green dotted lines, respectively. The black dash-dotted line $F_{M}^{(1)}\left(\gamma^{\text {tot }}\right)=2$ shows the RMT limit for very strong absorption. Inset (a) shows the dependence of the average transmission coefficient $T$ on the microwave frequency $\nu$. The internal absorption strength parameter $\gamma$ as a function of the average transmission coefficient $T$ is presented in inset (b). The values of the parameter $\gamma$ are given at the positions marked by empty diamonds.

matrix theory (RMT). One should note that the nearestneighbor spacing distribution $P(s)$ is less sensitive to missing levels than the power spectrum $\langle s(\tilde{k})\rangle$.

Figure $2 \mathrm{~b}$ shows the experimental distribution of the average power spectrum $\langle s(\tilde{k})\rangle$ (black diamonds) compared to theoretical prediction (red dash-dotted line). The fraction of the observed levels was unambiguously determined to $\varphi=0.94 \pm 0.02$, by comparison of the experimental power spectrum to the theoretical one. The experimental results are also compared to those for the eigenvalues of random matrices from the GOE (black solid line). The experimental results are in very good agreement with the theoretical ones, confirming that bow-tied shape microwave billiard exhibits spectral properties typical for chaotic systems with the fraction of observed levels $\varphi=0.94$.

In Fig. 3 the enhancement factor $F_{M}^{(1)}\left(\gamma^{\text {tot }}\right)$ of the twoport scattering matrix $\hat{S}(\nu)$ of the microwave quarter bow-tie cavity is shown for open channels $M=2$ (red full diamonds), $M=4$ (blue full triangles), $M=6$ (orange full circles), and $M=9$ (green full squares) as a function of the total absorption strength $\gamma^{\text {tot }}$. Due to significant fluctuations of the enhancement factor $F_{M}^{(1)}\left(\gamma^{t o t}\right)$ the experimental points were obtained by averaging of $F_{M}^{(1)}\left(\gamma^{\text {tot }}\right)$ over 100 different cavity realizations and the antennas positions in the moving frequency window $(\nu-\delta \nu / 2, \nu+\delta \nu / 2)$, where $\delta \nu=1.0 \mathrm{GHz}$. The theoretical results are shown in Fig. 3 for $M=2, M=4, M=6$, and $M=9$ channels with red, blue, orange, and green dotted lines, respectively. The experimental results are in good agreement with the theoretical ones. The black dash-dotted line $F_{M}^{(1)}\left(\gamma^{\text {tot }}\right)=2$ shows the RMT limit for very strong absorption. The inset (a) in Fig. 3 shows the dependence of the average transmission coefficient $T$ on microwave frequency $\nu$. The internal absorption strength parameter $\gamma$ depends on the microwave frequency and therefore also on the average transmission coefficient $T$ and was changed from 0.9 to 2.8 with the increase of the frequency $\nu$ from 6 to $12 \mathrm{GHz}$. The inset (b) in Fig. 3 presents the internal absorption strength parameter $\gamma$ as a function of the average transmission coefficient $T$. The values of the parameter $\gamma$ are given at the positions marked by empty diamonds.

\section{Summary}

The elastic enhancement factor $F_{M}^{(1)}\left(\gamma^{t o t}\right)$ for the microwave chaotic quarter bow-tie cavity in the presence of strong open channels $M$ and the total absorption strength $\gamma^{\text {tot }}$ was studied numerically and experimentally. The experimental results were obtained for $2 \leq M \leq 9$ open channels, moderate internal absorption strength $\gamma=0.9-2.8$, and the total absorption strength $\gamma^{\text {tot }}=1.6-11.6$. We showed that the experimental and theoretical results are close to each other. Moreover, the spectral properties of the microwave billiard with $M=2$ open channels were studied using missing level statistics such as the nearest-neighbor spacing distribution and the average power spectrum. This analysis revealed that the studied microwave billiard possesses time reversal symmetry and is fully chaotic. This finding is crucial for a comparison of the experimental results with the theoretical ones which are based on random matrix theory.

\section{Acknowledgments}

This work was supported in part by the National Science Centre, Poland, Grants Nos. UMO-2016/23 /B/ST2/03979 and UMO-2018/30/Q/ST2/00324. B.D. thanks the National Natural Science Foundation of China for financial support through Grants Nos. 11775100 and 11961131009.

\section{References}

[1] P.A. Moldauer, Phys. Rev. 123, 968 (1961); Phys. Rev. B 135, 642 (1964).

[2] W. Kretschmer, M. Wangler, Phys. Rev. Lett. 41, 1224 (1978).

[3] J.J.M. Verbaarschot, Ann. Phys. (New York) 168, 368 (1986).

[4] J.J.M. Verbaarschot, H.A. Weidenmüller, M.R. Zirnbauer, Phys. Rep. 129, 367 (1985). 
[5] Y. Kharkov, V. Sokolov, Phys. Lett. B 718, 1562 (2013).

[6] Y.V. Fyodorov, D.V. Savin, H.J. Sommers, J. Phys. A 38, 10731 (2005).

[7] D.V. Savin, Y.V. Fyodorov, H.J. Sommers, Acta Phys. Pol. A 109, 53 (2006).

[8] B. Dietz, T. Friedrich, H.L. Harney, M. Miski-Oglu, A. Richter, F. Schäfer, H.A. Weidenmüller, Phys. Rev. E 81, 036205 (2010).

[9] V. Sokolov, O. Zhirov Acta Phys. Pol. A 128, 990 (2015).

[10] C. Fiachetti, B. Michelson, Electron. Lett. 39, 1713 (2003).

[11] X. Zheng, S. Hemmady, T.M. Antonsen, Jr. S.M. Anlage, E. Ott, Phys. Rev. E 73, 046208 (2006).

[12] J.H. Yeh, Z. Drikas, J. Gil Gil, S. Hong, B.T. Taddese, E. Ott, T.M. Antonsen, T. Andreadis, S.M. Anlage, Acta Phys. Pol. A 124, 1045 (2013).

[13] M. Ławniczak, M. Białous, V. Yunko, S. Bauch, L. Sirko, Phys. Rev. E 91, 032925 (2015).

[14] M. Białous, B. Dietz, L. Sirko, Phys. Rev. E 100 012210 (2019).

[15] M. Ławniczak, S. Bauch, O. Hul, L. Sirko, Phys. Rev. E 81, 046204 (2010).

[16] M. Ławniczak, S. Bauch, O. Hul, L. Sirko, Phys. Scr. T143, 014014 (2011).

[17] M. Ławniczak, S. Bauch, O. Hul, L. Sirko, Phys. Scr. T147, 014018 (2012).

[18] M. Białous, V. Yunko, S. Bauch, M. Ławniczak, B. Dietz, L. Sirko, Phys. Rev. Lett. 117, 144101 (2016).

[19] M. Ławniczak, L. Sirko, Sci. Rep. 9, 5630 (2019)

[20] O. Hul, S. Bauch, P. Pakoński, N. Savytskyy, K. Życzkowski, L. Sirko, Phys. Rev. E 69, 056205 (2004).

[21] M. Ławniczak, J. Lipovský, L. Sirko, Phys. Rev. Lett. 122, 140503 (2019).

[22] M. Ławniczak, S. Bauch, L. Sirko, in: Handbook of Applications of Chaos Theory, Eds. Christos Skiadas, Charilaos Skiadas, CRC Press, Boca Raton 2016 p. 559 .

[23] O. Hul, M. Ławniczak, S. Bauch, A. Sawicki, M. Kuś, L. Sirko, Phys. Rev. Lett. 109, 040402 (2012).

[24] L. Sirko, P.M. Koch, R. Blümel, Phys. Rev. Lett. 78, 2940 (1997)

[25] H.J. Stöckmann, Quantum Chaos: An Introduction, Cambridge University Press, Cambridge 2000.
[26] Y. Hlushchuk, A. Błędowski, N. Savytskyy, L. Sirko, Phys. Scr. 64, 192 (2001).

[27] R. Blümel, P.M. Koch, L. Sirko, Found. Phys. 31 , 269 (2001).

[28] S. Hemmady, X. Zheng, E. Ott, T.M. Antonsen, S.M. Anlage, Phys. Rev. Lett. 94, 014102 (2005).

[29] B. Dietz, A. Richter, Chaos 25, 097601 (2015).

[30] R. Blümel, A. Buchleitner, R. Graham, L. Sirko, U. Smilansky, H. Walther, Phys. Rev. A 44, 4521 (1991).

[31] M. Bellermann, T. Bergemann, A. Haffmanns, P.M. Koch, L. Sirko, Phys. Rev. A 46, 5836 (1992).

[32] L. Sirko, S. Yoakum, A. Haffmans, P.M. Koch, Phys. Rev. A 47, R782 (1993).

[33] L. Sirko, P.M. Koch, Appl. Phys. B 60, S195 (1995).

[34] L. Sirko, A. Haffmans, M.R.W. Bellermann, P.M. Koch, Europhys. Lett. 33, 181 (1996).

[35] L. Sirko, S.A. Zelazny, P.M. Koch, Phys. Rev. Lett. 87, 043002 (2001).

[36] L. Sirko, P.M. Koch, Phys. Rev. Lett. 89, 274101 (2002).

[37] M. Krenn, X. Gu, A. Zeilinger, Phys. Rev. Lett. 119 240403 (2017).

[38] M.R. Brier, J.B. Thomas, A.M. Fagan, J. Hassenstab, D.M. Holtzman, T.L. Benzinger, J.C. Morris, B.M. Ances, Neurobiol. Aging 35, 757 (2014).

[39] P. Arrighi, S. Martiel, Phys. Rev. D 96, 024026 (2017).

[40] H.J. Stöckmann, J. Stein, Phys. Rev. Lett. 64, 2215 (1990).

[41] M. Ławniczak, M. Białous, V. Yunko, S. Bauch, L. Sirko, Phys. Rev. E 98, 012206 (2018).

[42] O. Bohigas, M.P. Pato, Phys. Rev. Lett. 595, 171 (2004).

[43] R.A. Molina, J. Retamosa, L. Muñoz, A. Relaño, E. Faleiro, Phys. Lett. B 644, 25 (2007).

[44] B. Dietz, V. Yunko, M. Białous, S. Bauch, M. Ławniczak, L. Sirko, Phys. Rev. E 95, 052202 (2017).

[45] A. Relaño, J.M.G. Gómez, R.A. Molina, J. Retamosa E. Faleiro, Phys. Rev. Lett. 89, 244102 (2002).

[46] E. Faleiro, J.M.G. Gómez, R.A. Molina, L. Muñoz, A. Relaño, J. Retamosa, Phys. Rev. Lett. 93, 244101 (2004). 